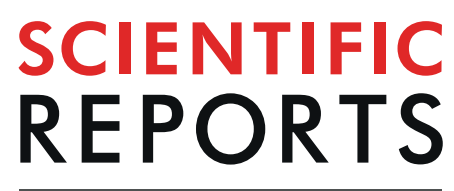

natureresearch

\title{
OPEN Apple B-box factors regulate light-responsive anthocyanin biosynthesis genes
}

Blue J. Plunkett ${ }^{1}$, Rebecca Henry-Kirk $\mathbb{1}^{1}$, Adam Friend $\mathbb{B}^{2}{ }^{2}$, Robert Diack ${ }^{2}$, Susanne Helbig ${ }^{1,3}$, Katriina Mouhu $\mathbb{1}^{1,4}$, Sumathi Tomes ${ }^{1}$, Andrew P. Dare ${ }^{1}$, Richard V. Espley ${ }^{1}$, Joanna Putterill ${ }^{5}$ \& Andrew C. Allan $\mathbb{1}^{1,5^{*}}$

Environmentally-responsive genes can affect fruit red colour via the activation of MYB transcription factors. The apple $B$-box (BBX) gene, BBX33/CONSTANS-like 11 (COL11) has been reported to influence apple red-skin colour in a light- and temperature-dependent manner. To further understand the role of apple $B B X$ genes, other members of the $B B X$ family were examined for effects on colour regulation. Expression of $23 B B X$ genes in apple skin was analysed during fruit development. We investigated the diurnal rhythm of expression of the $B B X$ genes, the anthocyanin biosynthetic genes and a MYB activator, MYB10. Transactivation assays on the MYB10 promoter, showed that BBX proteins 1, 17, $15,35,51$, and 54 were able to directly function as activators. Using truncated versions of the MYB10 promoter, a key region was identified for activation by BBX1. BBX1 enhanced the activation of MYB10 and MdbHLH3 on the promoter of the anthocyanin biosynthetic gene DFR. In transformed apple lines, over-expression of BBX1 reduced internal ethylene content and altered both cyanidin concentration and associated gene expression. We propose that, along with environmental signals, the control of $M Y B 10$ expression by BBXs in 'Royal Gala' fruit involves the integration of the expression of multiple $B B X$ s to regulate fruit colour.

Apple is an important fruit crop, both commercially and from a dietary nutrition perspective. Compounds found in apple have been shown to have positive effects on human health ${ }^{1,2}$. These compounds are derived from secondary metabolites and comprise a diverse set of bioactive phytochemicals including phenylpropanoids, which contain the flavonoid subclass that includes anthocyanins, the pigments responsible for the red colour of apple. Potential health benefits include anti-inflammatory, anti-carcinogenic, and antioxidant properties ${ }^{3,4}$. Fruit colour is also a strong market driver, with consumer preferences varying between countries and regions ${ }^{5,6}$. In general, consumer appeal tends towards red colour, with red apples being preferred to yellow or green cultivars ${ }^{5-7}$. Apple colour and patterning varies naturally within and between cultivars, and in many apple cultivars this is influenced by environmental conditions, modulating the concentrations of chlorophyll, carotenoid, and anthocyanin ${ }^{8,9}$. The red colour of apple skin is composed almost exclusively of the anthocyanin derivative, cyanidin-3-O-galactoside.

Anthocyanin is important for plants as it protects against photo-oxidative and heat damage caused by sunlight, as well as influencing pollination and seed dispersal ${ }^{10-12}$. The accumulation of anthocyanins in plants appears to be linked to biotic and abiotic stress, with anthocyanin concentrations being increased in response to herbivory, fungal and viral pathogens, wounding, temperature, high light and UV, mineral imbalance, drought, salinity, anoxia, ozone, herbicides ${ }^{13}$. Research on the regulation of anthocyanin production has identified both the biosynthetic pathways genes ${ }^{14-16}$ and the major regulating transcription factors (TFs), comprising the MYB-bHLH-WD40 (MBW) complex ${ }^{17}$. This complex binds promoter regions of anthocyanin biosynthetic genes and MYB10 and increases transcription, as shown for Arabidopsis (Arabidopsis thaliana $)^{17}$, nectarine ${ }^{18}$, petunia

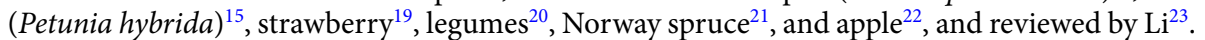

The MYBs that regulate anthocyanin accumulation in apple have been well studied ${ }^{22,24-26}$. Alleles MYBA and $M Y B 1$ are identical and share $98 \%$ sequence homology with $M Y B 10$, which differs by three amino acids in the

${ }^{1}$ The New Zealand Institute for Plant and Food Research Limited (PFR), Mt Albert, Private Bag 92169, Auckland, New Zealand. ${ }^{2}$ PFR, 55 Old Mill Road, RD 3, Motueka, 7198, New Zealand. ${ }^{3}$ BIOTECON Diagnostics GmbH, Hermannswerder 17, 14473, Potsdam, Germany. ${ }^{4}$ Faculty of Agriculture and Forestry, University of Helsinki, Helsinki, Finland. ${ }^{5}$ School of Biological Sciences, University of Auckland, Private Bag 92019, Auckland, New Zealand. *email: Andrew.Allan@plantandfood.co.nz 
open reading frame ${ }^{25}$. The R2R3 binding region of these and anthocyanin-related MYBs in other plant species is highly conserved ${ }^{25}$. In apple, MYB10, bHLH3/33 and a WD40 protein TTG1 have been shown to form the MBW complex seen in other species to regulate anthocyanin concentrations ${ }^{26}$. Patterning of apple fruit skin includes striped and blushed, and this variation can be explained by the differential expression of MYB10 and degree of methylation in the promoter ${ }^{27}$.

Repressors also play an important role in the regulation of anthocyanin production. The genes involved and the mechanisms by which they operate are beginning to be resolved. For example, two repressors have been reported in petunia, MYB27 and MYBx ${ }^{15,28}$. MYB27 is a R2R3 type MYB, which can interfere with the MBW complex by preventing its formation or being incorporated, and converting the complex into a repression complex. MYBx is a R3 type MYB that also appears to compete for interaction with the bHLH component of the MBW complex. MYB27 contains an EAR (ethylene-responsive element binding factor associated amphiphilic repression domain) and TLLLFR motif, both of which appear to confer the ability to repress anthocyanin ${ }^{15}$. MYBL2, an R3-MYB, has been identified in Arabidopsis as a repressor of anthocyanin and also contains the TLLLFR motif ${ }^{29,30}$. Similar repression mechanisms have also been identified in strawberry ${ }^{31}$. In apple, a family of MYB repressors of anthocyanin were identified after heat treatment and were characterised by the EAR motif ${ }^{32}$.

Repression and activation of the anthocyanin pathway is responsive to environmental signals. Lin-Wang et $a l .{ }^{32}$ have shown that heat reduces apple skin MYB10 expression, while Xie et al. ${ }^{33}$ showed that cold induces bHLH3 to bind with MYB1 and upregulate the promoters of anthocyanin biosynthetic genes. Appropriate light conditions are also required for anthocyanin accumulation in many apple cultivars ${ }^{34,35}$ and anthocyanin has been shown to increase in response to ultraviolet-B (UV-B) irradiation and to absorb UV- $\mathrm{B}^{7,36}$. Long-term changes, enduring after six weeks of postharvest storage, were found in apples grown on trees covered by a canopy of UV-reducing film ${ }^{37}$. The reduction of UV affected a wide range of fruit qualities, including a delay in ripening, reduction in fruit size, and decrease of anthocyanin and flavonol concentrations. Many of the transcription factors responsible for these environmentally induced anthocyanin changes have been identified. As a consequence of these genes being responsive to environmental signals they can exhibit expression rhythms that follow the stimulus. These rhythms can be detected by measuring transcription. In Arabidopsis, ELONGATED HYPOCOTYL 5 (HY5), a basic leucine zipper (bZIP) transcription factor, receives signals from photoreceptors and regulates anthocyanin by directly binding promotors of PAP1 (AtMYB75) and anthocyanin biosynthetic genes ${ }^{38}$. HY5 is also involved in low temperature-induced anthocyanin accumulation ${ }^{39}$. LIGHT-REGULATED ZINC FINGER PROTEIN 1 (LZF1, or B-BOX DOMAIN PROTEIN 22) has been found to act downstream of HY5 to inhibit anthocyanin ${ }^{40}$. The photoreceptor UV RESISTANCE LOCUS 8 (UVR8) provides light-signalling control of various pathways, including regulating HY5 and interacting with CONSTITUTIVE PHOTOMORPHOGENIC 1 $(\mathrm{COP} 1)^{41,42}$. COP1 is a RING finger type ubiquitin E3 ligase involved in the targeted degradation of various anthocyanin influencing factors, including MYB1 in apple ${ }^{43-45}$.

Although many transcription factors have been identified in the regulation of anthocyanin biosynthesis, a full understanding of how light regulates this process is still being developed. The role BBXs play is an example. Studies focusing on specific members of the BBX gene family in apple have shown that UV-B light in particular upregulates MdCOL11/BBX33 expression in a temperature-dependent manner ${ }^{7} . M d B B X 33$ is a close homolog of AtBBX22/LZF1, also known as STH3 (Salt Tolerance Homolog 3) and DBB3 (Double B-Box zinc finger 3) ) $^{7,40,46}$. Bai et al. ${ }^{7}$ generated transgenic lines of Arabidopsis overexpressing apple BBX33, which had increased anthocyanin accumulation in seedlings and showed also that the expression profiles of BBX33 and MYB10 are related during different temperature and light regimes. Using dual luciferase assays it was shown that BBX33 upregulates the MYB10 promoter. These findings suggest that BBX33 functions downstream of HY5 and upstream of MYB10 as a component in the environmental sensing pathway, which leads to anthocyanin production in apple.

Discovery of the first $B B X$, in Arabidopsis, came from characterising the flowering pathway. CONSTANS (CO/BBX1) was shown to be important for regulating transcription levels of floral integrators where it is a key factor controlling photoperiodic flowering response in Arabidopsis ${ }^{47-51}$. CO/BBX1 was subsequently identified as a member of the larger $B B X$ gene family. This is a family characterised by the proteins they encode possessing two highly conserved domains, an N-terminal zinc finger BBX ${ }^{52,53}$ and a C-terminal CCT (CO, CO-like, TIMING OF CAB EXPPRESSION 1) domain ${ }^{54-56}$. Both domains are involved with mediating transcriptional regulation and protein-protein interaction. Arabidopsis has $32 B B X$ genes (1-32), which fall into five groups (I-V) based on common variations in the conserved BBX and CCT domains $s^{53}$. Twenty-one of the 32 AtBBX gene sequences contain two BBXs in tandem, the remaining 11 contain one $B B X^{55,57}$. This classification of $B B X$ genes using number of conserved motifs has been reiterated by Huang et al. ${ }^{58}$ in rice. Further analysis, which included comparison of BBX sequences across a diverse range of species, has been conducted by Crocco and Botto ${ }^{53}$ where 214 BBX proteins were identified and compared, providing evolutionary insight into these genes.

More recently, Liu et al..$^{59}$ reported a comprehensive list of the apple $B B X$ gene family. A total of $64 M d B B X$ s were identified. The phylogeny and motif structure were reported, along with chromosomal locations. Although naming these genes as 'COLs' (for CONSTANS-LIKE) has been reported ${ }^{56,60-64}$, Liu et al. ${ }^{59}$ and others ${ }^{53,55}$ selected ' $B B X$ ' as the convention so as to include genes that do not contain the 3' CCT motif, and to align with the naming convention reported by Khanna et al.$^{57}$ in Arabidopsis.

Here we examined members of the $B B X$ gene family in apple, using the naming convention from Liu et al. ${ }^{59}$, with an emphasis on potential roles in anthocyanin regulation. The expression of $23 B B X$ s, representing the five sub-clades in the gene family, in Malus $\mathrm{x}$ domestica 'Royal Gala' fruit skin during fruit development was assessed. Given that many BBX genes, including Arabidopsis CO,COL1 and COL2, show diurnal patterns of expression ${ }^{64,65}$, a detailed examination of diurnal expression patterns in both candidate apple BBX genes and anthocyanin structural genes was performed. Expression profiles of anthocyanin biosynthetic genes and the MYB regulator were tested for expression correlations. A subset of the BBX proteins were screened for their ability to activate the promoter of $M Y B 1 / 10$, which was also tested for any dependent BBX-related motifs. Furthermore, the activation 
of the promoter of the anthocyanin biosynthetic gene DIHYDROFLAVONOL 4-REDUCASE (DFR) by MYB10 and bHLH3 was assessed in the presence of BBX1. Finally, we generated and characterized five transgenic lines of apple trees overexpressing $B B X 1$ and measured postharvest metrics and metabolite content differences compared to wildtype.

\section{Materials and Methods \\ Plant material. Apple fruit tissue used for the development series was sampled from 'Royal Gala' trees grown in standard conditions in a glasshouse in 2012/2013 at seven time points during development: $35,65,85,110$, 120,130 and 140 days after full bloom (DAFB) at $1300 \mathrm{~h}$. Full bloom occurred on 28 September 2012 and at each time point five fruit were harvested and pooled. Apple fruit tissue used for diurnal sampling were collected from 'Royal Gala' apple trees in the Plant and Food Research orchard, Motueka, Nelson, New Zealand, on 8-10 February 2011 (season one) and from the Plant and Food Research orchard, Mt Albert, Auckland, New Zealand, on 27 February - 2 March 2012 (season two). Apple leaf tissue was harvested at the same time as fruit using five pooled leaves per time point. Fruit sampling began at 120 DAFB. Due to length of time course, number of fruit harvested per time point had to be limited to three fruit at each time point, which occurred at $1300 \mathrm{~h}$, and then pooled. To help with this, two seasons were sampled. Apple trees over-expressing BBX1 were grown in the Plant and Food Research glasshouse in Auckland. Three pooled fruit were used for each replicate and run in quadrupli- cate for gene expression analysis and the average reading for five fruit is presented for ethylene, weight, firmness, and soluble solids content (measured as ${ }^{\circ} \mathrm{Brix}$ ). High performance liquid chromatography (HPLC) and liquid chromatography-mass spectrometry (LCMS) data are also presented as the average reading from five fruit. For each fruit all of the skin was removed using a scalpel and immediately frozen in liquid nitrogen along with leaf samples, ground to powder without thawing, then stored at $-80^{\circ} \mathrm{C}$ until RNA extraction for RT-qPCR analysis.}

Promoter and gene cloning. Full-length coding sequences of $M Y B 10, M Y B 8, b H L H 3, b H L H 15$ and $B B X 1$, $9,10,15,17,33,35,37,42,43 / 44,47,51,54,57$ were isolated from 'Royal Gala' cDNA and inserted into the pGEM-T Easy vector (Promega, Madison, USA) before being inserted into the pHEX2 binary vector via restriction site cloning ${ }^{66}$. Promoter sequences for the $\mathrm{R}_{1}$ allele of $M Y B 10, D F R, B B X 1$, and $B B X 15$ were isolated from 'Royal Gala' genomic DNA and inserted into the cloning site of pGreenII08005'-LUC as previously described by Espley et al. ${ }^{67}$. Truncated versions of the promotor of $M Y B 10(\Delta \mathrm{a}$ and $\Delta \mathrm{b})$ were generated by designing primers to amplify $834 \mathrm{bp}$ and $405 \mathrm{bp}$ fragments upstream of the MYB10 start site. Primer sequences are given in Supplementary Table 1.

Dual luciferase assay of transiently transformed Nicotiana benthamiana leaves. Nicotiana benthamiana plants were used for transient transformation assays. These plants were grown at Plant and Food Research, Auckland, according to standard glasshouse management practices. Agrobacterium tumefaciens GV3101 was transformed by electroporation to introduce constructs. $N$. benthamiana leaves were transiently transformed for the dual luciferase assay as previously reported by Lin-Wang et al. ${ }^{68}$.

Metabolite analysis. Anthocyanin content was measured by HPLC. For the more comprehensive analysis of the $B B X 1$ over-expressing lines, HPLC was used to detect anthocyanins and polyphenols. Ground apple skin tissue $(100 \mathrm{mg})$ was freeze-dried for 12 hours. Acidified $(0.5 \% \mathrm{HCL})$ methanol ( $5 \mathrm{x}$ volume) was added to the tissue and placed in the dark for 3 hours to extract. Samples were centrifuged at 16,000 rpm for 4 minutes, then supernatant transferred to a new tube for vacuum evaporation. Extract was then re-suspended in $500 \mu \mathrm{L}$ of $20 \%$ methanol, filtered into a new tube and vacuum evaporated. Extract was then finally re-suspended in $20 \mu \mathrm{L}$ methanol for analysis. Extract was analysed on a Dionex Ultimate 3000 RS system as previously described by Dare et al. ${ }^{69}$.

Gene expression analysis by RT-qPCR. RNA was isolated using Spectrum ${ }^{\mathrm{TM}}$ RNA extraction kit (Sigma-Aldrich) according to the manufacturer's instructions. RNA quantification was performed using a NanoDrop ${ }^{\text {TM }}$ ND-1000 UV-Vis Spectrophotometer (NanoDrop Technologies, Thermo Scientific, USA) and visualized by gel electrophoresis. First strand cDNA synthesis of $2 \mu \mathrm{g}$ total RNA was performed using Qiagen QuantiTect ${ }^{\circledR}$ Reverse Transcription Kit (Qiagen). Expression analysis was performed using four technical replicates on a Roche LightCycler ${ }^{\circledR} 480$ platform and LightCycler FastStart SYBR Green I Master Mix as described previously ${ }^{22}$. Data was analysed using the Target/Reference ratio calculated with the LightCycler $480{ }^{\circledR}$ software version 1.5.0.39 (Roche) and primer efficiency corrections added. CP values for housekeeper, Actin, did not vary by more the 1.72 cycles during the $48 \mathrm{~h}$ and $52 \mathrm{~h}$ of sampling for season one and two respectively. Primers were designed to optimise efficiency as previously described ${ }^{32}$. Primer sequences are given in Supplementary Table 1.

Internal ethylene assessment. Internal ethylene concentration of apple fruit was determined using a needle and syringe to extract gas from the internal core cavity of attached fruit during season 2 of sampling at 130 $\mathrm{DAFB}$ at $1300 \mathrm{~h}$. BBX1 over-expressing lines samples were taken at $125 \mathrm{DAFB}$ at $1300 \mathrm{~h}$. Core cavity gas $(1 \mathrm{~mL})$ was injected into a gas chromatograph (Hewlett Packard, 5890 series II) and run according to the method of Johnston et al..$^{70}$.

Statistical analysis. To test correlations between genes, the gene expression data were used to generate correlation coefficient values using two-sided Pearson's product-moment correlation in R version 3.2.4 ${ }^{71}$. Differences between averages were analysed using Analysis of Variance (ANOVA) in GenStat version 17.1.0.14713. Post hoc pairwise differences were determined using Fisher's LSD test at the 5\% significance level ${ }^{72}$. 

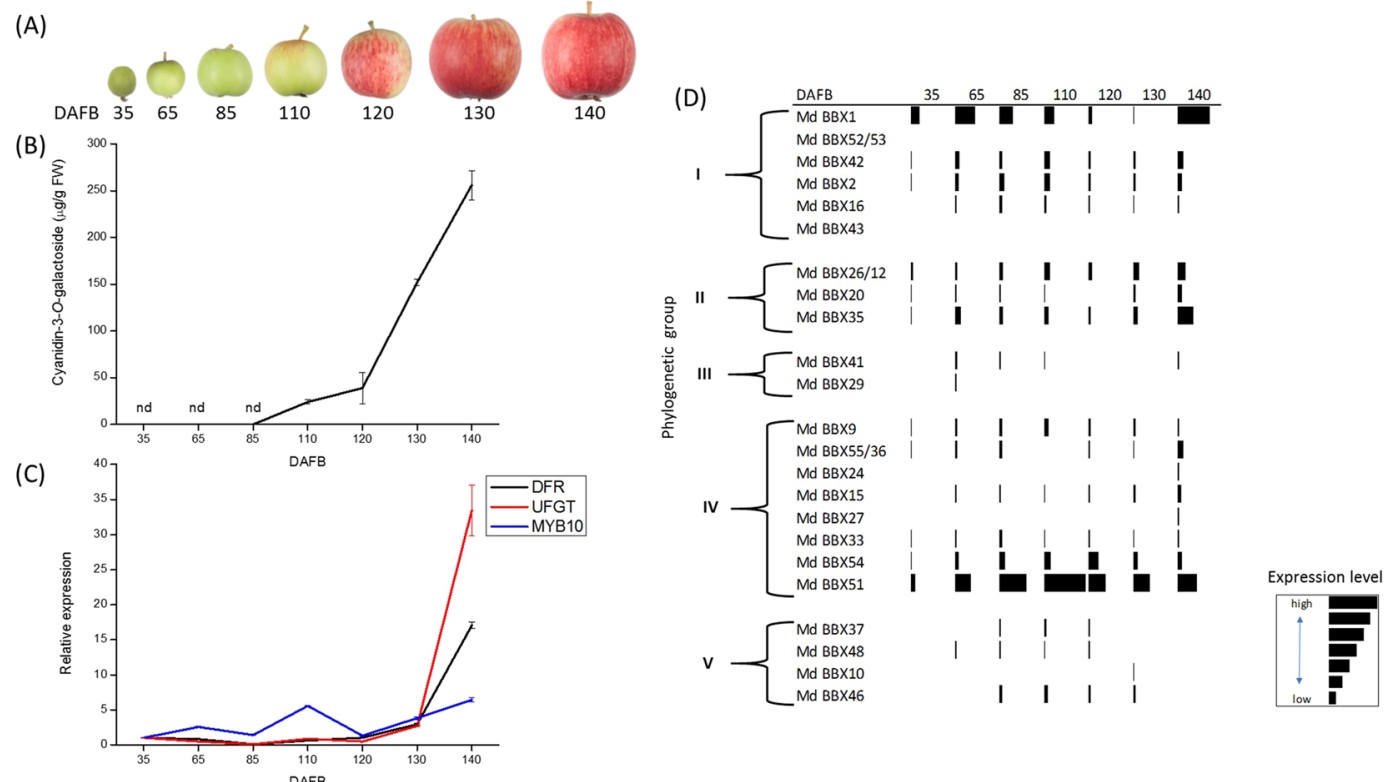

Figure 1. Assessment of skin anthocyanin accumulation during apple fruit development compared with $B B X$ gene expression. Apple fruit were assessed at seven time points; 35, 65, 85, 110, 120, 130, and 140 DAFB. (A) Photographs of representative apple fruit at each stage of development assessed showing accumulation of red colour. (B) Cyanidin-3-O-galactoside accumulation in apple fruit skin at seven time points during development. Values are the average concentration of cyanidin as determined by HPLC from two pooled fruit per time point. Time points where no cyanidin was detected are indicated by 'nd'. Error bars show SE of four technical replicates. (C) Relative expression of DFR, UFGT, and MYB10 in apple fruit skin. Expression is relative to Actin normalized to 35 DAFB. Error bars show SE of four technical replicates. (D) Table showing relative expression level of selected $B B X s$ in apple fruit skin at seven time points during development. Phylogenetic groups reported by Liu et al..$^{59}$ indicated. Black bar at each DAFB indicates level of expression relative to the other $B B X$ genes. Key shows that the length of the black bars indicates level of $B B X$ expression relative to Actin and normalised to highest expressed $B B X$ (BBX51 at $110 \mathrm{DAFB})$.

Stable transformation of apple. The cDNA sequence of apple $B B X 1$ was cloned into the binary vector pSAK $277^{22}$ driven by the $35 \mathrm{~S}$ promoter and transferred into Agrobacterium strain LBA4404. Transgenic lines of 'Royal Gala' plants were generated by Agrobacterium-mediated transformation of leaf pieces using a method previously reported by Yao et al. ${ }^{73}$. Transgenic plants were rooted and grown in a containment glasshouse at The New Zealand Institute for Plant and Food Research Limited (PFR), Auckland, New Zealand, with flowering and fruiting occurring three years after planting.

\section{Results}

Anthocyanin concentration and gene expression of the $B B X$ family in fruit skin during development. Apple fruit were assessed at seven time points during development: 35, 65, 85, 110, 120, 130, and 140 DAFB (Fig. 1A). At each time point, concentrations of cyanidin-3-O-galactoside, the expression of apple anthocyanin biosynthetic genes DFR and URIDINE DIPHOSPHATE (UDP)-GLUCOSE FLAVONOID 3-O-GLYCOSYLTRANSFERASE (UFGT), and the expression of anthocyanin-regulating transcription factor $M Y B 10$, as well as that of 23 genes from the $B B X$ family were measured. Cyanidin-3-O-galactoside content was detectable at 110 DAFB, increasing up to 140 DAFB (Fig. 1B). Expression of DFR, UFGT, and MYB10 increased during development, with the highest expression for all three genes seen at 140 DAFB (Fig. 1C). Transcripts of a subset of the $B B X$ family genes, representing all five sub clades ${ }^{59}$, were also examined in apple skin during fruit development (Fig. 1D). This identified BBXs with expression patterns that coincided with anthocyanin accumulation in developing fruit. The highest and lowest expression for the genes varied over the six time points measured within and between the different groups of $B B X$ s. Transcript abundance of many of the $B B X$ s started low and then increased during fruit development (data presented at higher resolution in Supplementary Fig. 1). BBX51 and $B B X 1$ were the two most highly expressed $B B X$ s examined. Expression of $B B X 51$ was highest at 110 DAFB, while $B B X 1$ had an expression peak early in development (65 DAFB) and then again at 140 DAFB. Some genes, such as $B B X 15$, were relatively lowly expressed but still displayed increasing expression during fruit development (Supplementary Fig. 1). Overall, BBXs from groups I, II and III had variable patterns of gene expression in the skin during fruit development, with the highest expression occurring early, middle, or late in the sampling. Group IV $B B X$ s generally displayed their lowest expression at 35 DAFB, with the exception of $B B X 27$ (Supplementary Fig. 1). Most group V $B B X$ s started with low expression and peaked at different times around the mid-range of the sampling period. 
Measuring diurnal patterns of transcription in apple leaves and fruit. Having demonstrated that the transcription of certain $B B X$ genes increases during fruit development in a similar pattern to the concentration of anthocyanin and key anthocyanin-related genes, we then tested the transcript abundance of selected genes during a diurnal time series at $120 \mathrm{DAFB}$. This is the stage at which anthocyanin is rapidly accumulating. To confirm that variation in expression could be detected in apple diurnally, we tested transcription of apple GIGANTEA (GI) in leaf and fruit tissue (Fig. 2). GI was selected as a positive control because of its role in the circadian pathway and its known pattern of expression ${ }^{74,75}$. GI was found to be expressed in a highly regular pattern (high in the evening and low in the morning) in accordance with findings in Arabidopsis ${ }^{74,75}$. The expression of BBX1 showed a distinct pattern of expression in the apple leaf, with the highest expression levels around $0400 \mathrm{~h}$ and lowest in the evening. Other genes, such as $B B X 51, D F R, H Y 5$, and $C O P 1$ also exhibited daily transcript fluctuations in apple leaves (Supplementary Fig. 2).

Diurnal rhythms of gene expression were assessed in fruit skin over two seasons and two locations, to control for environmental and seasonal variation (Fig. 2). GI expression in skin showed a diurnal expression rhythm with the highest expression in the evening at $2000 \mathrm{~h}$ and lowest at $0800 \mathrm{~h}$ for both seasons and sites, as was seen in leaves. $B B X 1$ peaked at $0400 \mathrm{~h}$ and was lowest in the evening, as was seen in leaves. A selection of light and temperature genes were also measured in apple skin to ascertain if detection of daily rhythms for genes reported to be involved in light and temperature perception was also possible in apple skin. These included COP1, HY5, and UVR8 involved in light response (Fig. 2), and CBF2 and HSFA2 $a$ involved in temperature-regulated expression (Fig. 2) 7,777 $^{7}$. A further six genes were tested (Supplementary Fig. 3). COP1 and HY5 expression patterns were similar, displaying peaks during daylight. These findings for apple COP1 support work in Arabidopsis where COP1 expression is high in the afternoon ${ }^{43}$. Peaks in the expression of UVR8 coincided with light periods except for the first night of season 2. As expected, CBF2 expression was elevated at night and HSFA2a was highest during the day (Fig. 2).

$B B X$ s 51, 33, and 15 were also selected for assessment (Fig. 2). BBX51 was chosen as it showed the highest expression during development (Fig. 1D), BBX33 because of its involvement in anthocyanin accumulation ${ }^{7}$, and $B B X 15$ as it showed increased transcription over fruit development (Fig. 1D). Expression of $B B X 51$ was highest in the morning and lowest in the evening. Expression of $B B X 33$ was not found to be as rhythmic as the other $B B X$ genes tested for season 1 , and in season 2 it showed highest expression in the evening and lowest in the morning. $B B X 15$ was also highest in the evening and lowest in the morning, for both seasons tested.

Correlation analysis of MYB10 and anthocyanin pathway genes. To further investigate diurnal gene expression in apple fruit skin tissue, we analysed the expression of anthocyanin structural genes PHENYLALANINE AMMONIA LYASE (PAL), CHALCONE SYNTHASE (CHS), CHALCONE ISOMERASE (CHI), FLAVANONE 3-HYDROXYLASE (F3H), DFR, LEUCOANTHOCYANIDIN DIOXYGENASE/ ANTHOCYANIDIN SYNTHASE (LDOX/ANS), UFGT, and the MYB10 TF (Fig. 3A). All the biosynthetic genes have a similar diurnal expression pattern to $M Y B 10$, with correlation analysis confirming a positive linear relationship, with four of the seven genes tested being statistically significant $(p<0.05)$ (Fig. 3B). However, no direct diurnal correlation with MYB10 and BBX transcripts was found (Supplementary Table 2), even when taking into account a possible delay in the correlation by analysing for the effect on MYB10 with a four- or eight-hour delay (data not shown).

Activation of the MYB10 promoter by $B B X$ genes. Members of the apple $B B X$ gene family were selected for promoter activation assays based on their expression during development (Fig. 1D and Supplementary Fig. 1). These $14 B B X$ genes were screened for activation of the MYB10 promoter using the dual luciferase system in $N$. benthamiana. The MYB10 promoter was infiltrated alone, or co-infiltrated with MYB10 +/- MdbHLH3 (Fig. 4). Compared with the controls, $B B X 1$ alone activated the MYB10 promoter and showed an additive affect when co-infiltrated with MYB10, but no further activation was found when $b H L H 3$ was added. BBX51 and BBX54 both activated the promoter when co-infiltrated with $M Y B 10$, but this effect was largely lost after the addition of $b H L H 3$. BBX15 did not activate the promoter alone; however, the promoter was activated by the addition of $M Y B 10$ and $M Y B 10$ plus $b H L H 3$. The highest activation of the promotor was when $B B X 35$ was co-infiltrated with $M Y B 10$ and $b H L H 3$. From these data, we identified $B B X 1,15,35,51$ and 54 as $B B X$ s capable of activating the promoter of MYB10. Furthermore, the addition of the $b H L H$ co-factor gene displayed all three possible effects, depending on the $B B X$ : no change, or decreased or increased activation.

Deletions in the $M Y B 10$ promotor reveal specific sites required for BBX1 activation. To further characterise the activation of the MYB 10 promoter by $B B X$ genes, we generated two truncated versions of the 1704 bp promoter sequence of $M Y B 10$ (Fig. 5A), MYB10 $a$ and $M Y B 10 \Delta b$ at $834 \mathrm{bp}$ and $405 \mathrm{bp}$, respectively, upstream of the ATG start site. We assessed the promoter sequence for cis-elements which might facilitate interaction with BBXs and found CCAAT motifs present. In yeast these motifs are bound by HEME ACTIVATOR PROTEINS (HAP), also known as NUCLEAR FACTOR Y (NF-Y) proteins, facilitated by domains similar to the CCT domain of many plant $B B X$ s in plants, including $B B X^{78,79}$. Due to some $B B X s$ containing the CCT domain it is possible they will bind the same CCAAT motif as the HAP proteins. The full MYB10 promotor sequence contains three CCAAT motifs, MYB10 $a$ contains one, while $M Y B 10 \Delta b$ contains none. The infiltration of $B B X 1$ alone resulted in a slight activation of MYB10 and MYB10A $a$ compared with MYB10 or bHLH3 controls (Fig. 5B). Most notably, the ability for the promoter to be activated by MYB10 and BBX1 dropped significantly for the $\Delta b$ promotor sequence (Fig. $5 \mathrm{~B}$ ). This also applied if bHLH3 was present. Apple MYB8 was included as a control to show specificity of the MYB10 infiltration co-factor to $B B X 1$ activation of the promoter ${ }^{22}$. 
GI - Leaf season 2

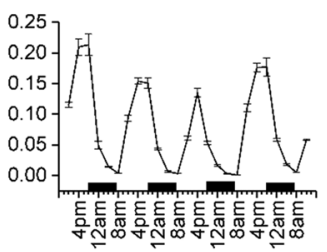

BBX1 - Leaf season 2

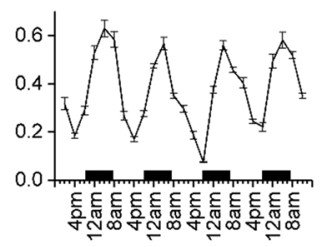

COP 1 - season 1
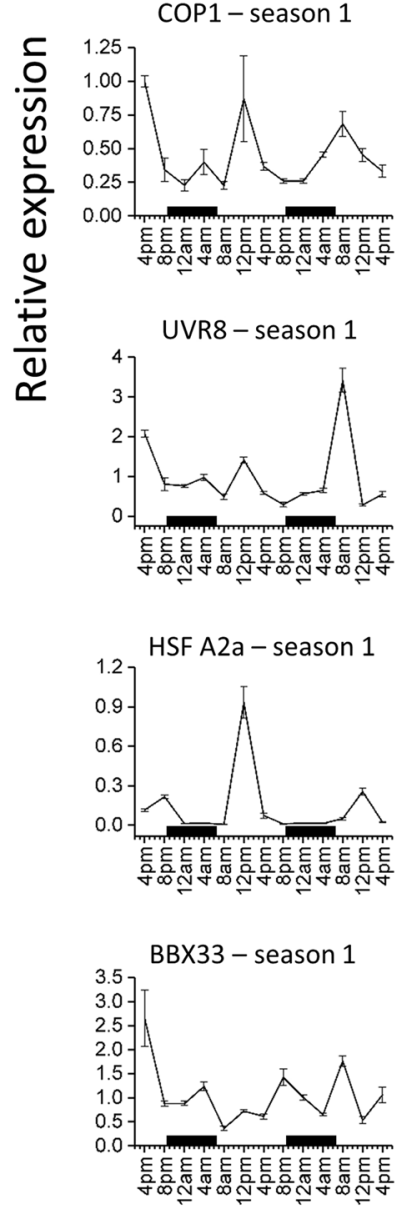

GI - Fruit season 1

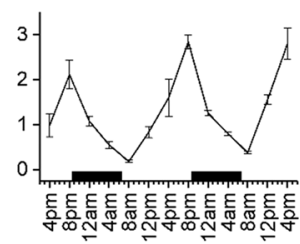

BBX1 - Fruit season 1

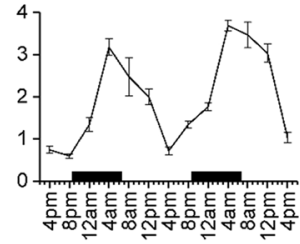

COP1 - season 2

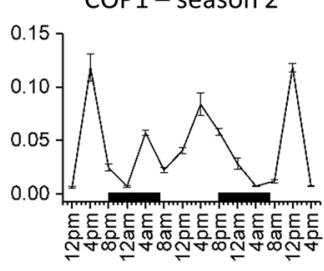

UVR8 - season 2

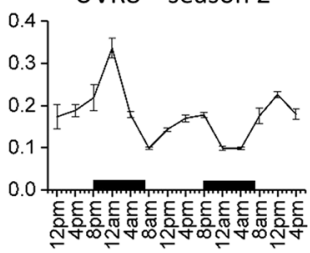

HSF A2a - season 2

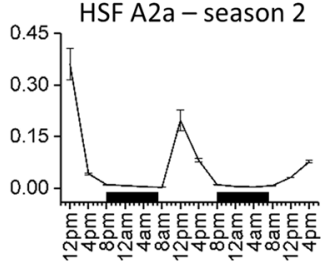

BBX33 - season 2

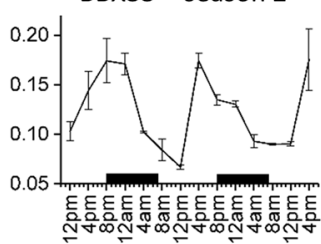

GI - Fruit season 2

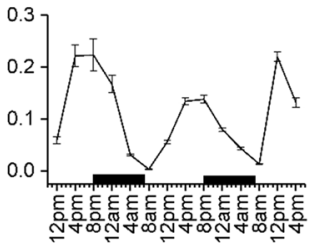

BBX1 - Fruit season 2

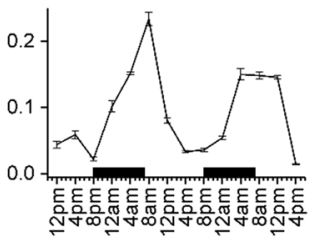

HY5 - season 1
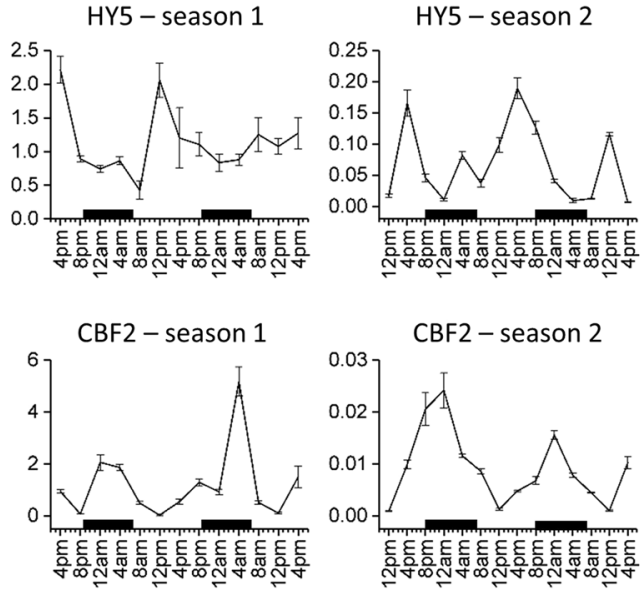
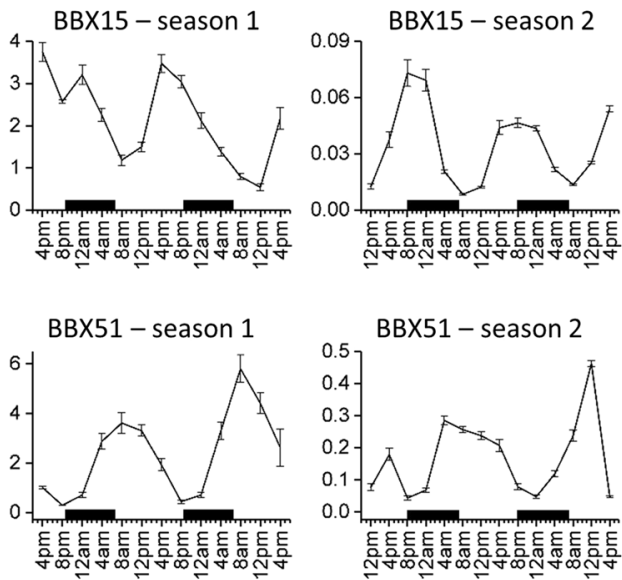

BBX51 - season 2

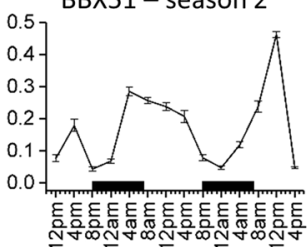

Time

Figure 2. Diurnal gene expression levels at four hour intervals over two seasons. Gene expression for apple Gigantea (GI) and BBX1 in apple leaf tissue and fruit skin tissue labelled, all other genes are fruit skin results. Other genes tested were light-associated COP1, HY5, and UVR8, temperature-linked genes CBF2 and HSFA2a and BBX genes 15, 33, and 51 from BBX group IV over two seasons. Expression results were normalised to Actin and error bars are SE of four technical replicates. Daylight hours were from $0640 \mathrm{~h}$ to $2040 \mathrm{~h}$ for season 1 and from $0700 \mathrm{~h}$ to $2000 \mathrm{~h}$ for season 2, rounded to the nearest $10 \mathrm{~min}$. Black bars indicate night.

The apple DFR promoter is activated by BBX1. Having demonstrated that some BBXs activated the MYB10 promoter (Fig. 4), the promoter of a gene encoding an anthocyanin biosynthetic enzyme, DFR, was selected to test for activation by BBX1. The promoter of DFR was selected as it displayed the most significant 


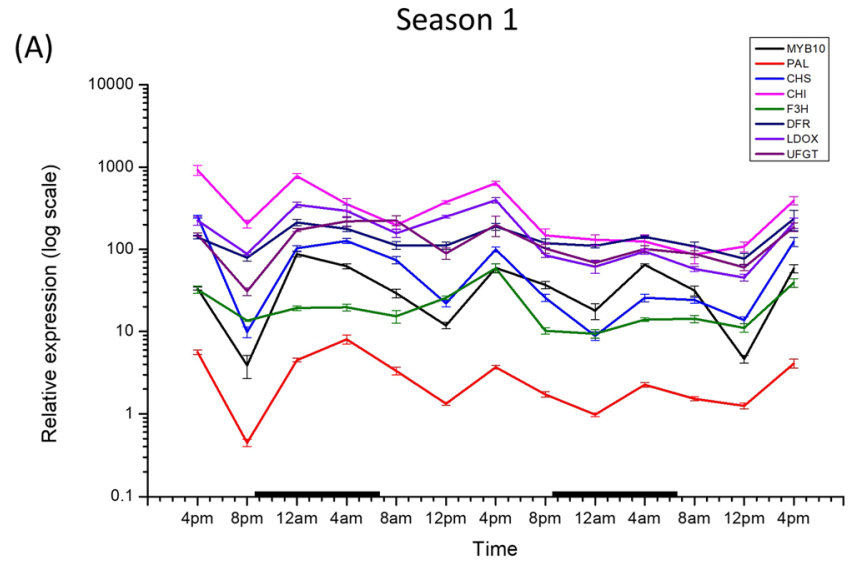

(B)

\begin{tabular}{cccc}
\hline Gene & cor & p-value & \\
\hline PAL & 0.6454 & 0.0172 & $*$ \\
\hline CHS & 0.4288 & 0.1437 & \\
CHI & 0.4615 & 0.1124 & \\
\hline F3H & 0.3618 & 0.2244 & \\
DFR & 0.8711 & 0.0001 & $* * *$ \\
LDOX & 0.6261 & 0.0221 & $*$ \\
UFGT & 0.6660 & 0.0130 & $*$ \\
\hline MYB10 & 1 & & \\
\hline
\end{tabular}

Figure 3. (A) Diurnal gene expression at four-hour intervals for $M Y B 10$ and seven anthocyanin biosynthetic genes (PAL, CHS, CHI, F3H, DFR, LDOX, and UFGT). Expression was normalised to Actin and error bars are SE of four technical replicates. Daylight hours were $0640 \mathrm{~h}$ to $2040 \mathrm{~h}$, rounded to the nearest $10 \mathrm{~min}$. Black bars indicate night. (B) Table of Pearson's product-moment correlation coefficient values for seven anthocyanin biosynthetic genes compared to MYB10 ( $p$-value $\left.<0.05^{*},<0.01^{* *},<0.001^{* * *}\right)$.

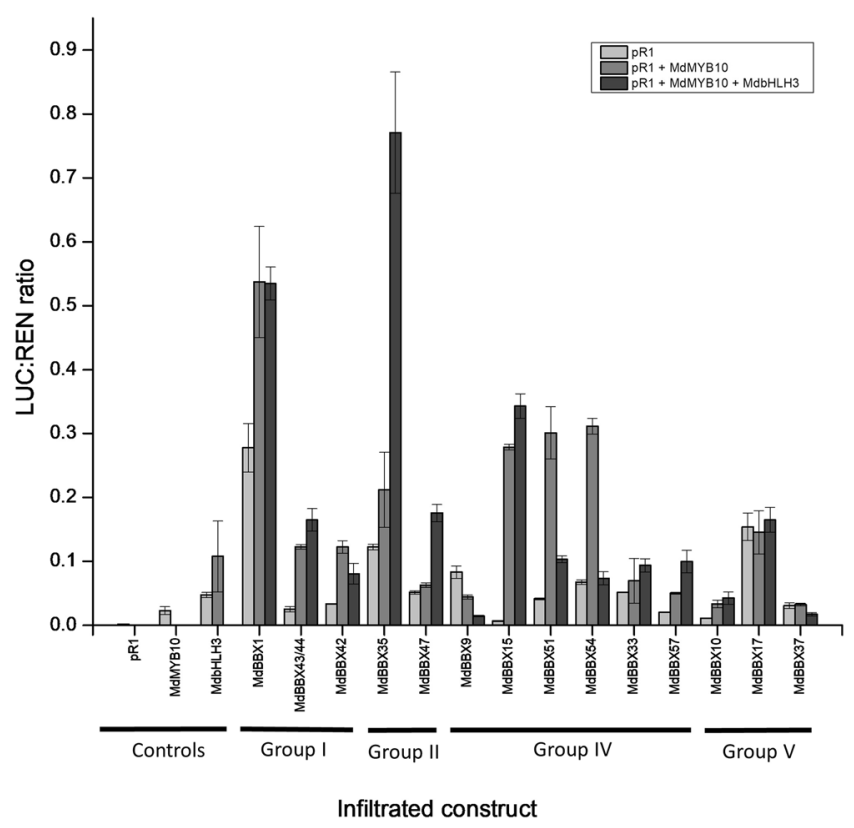

Figure 4. Transient expression assay of apple BBXs effect on the promoter of apple $M Y B 10$ in tobacco. $\mathrm{pR}_{1}$ denotes the MYB10 promoter to distinguish it from the MYB10 protein. Each BBX was infiltrated with pR1 (light grey bars), with pR1 and MYB10 (medium grey), and with pR1, MYB10, and bHLH3 (dark grey).

Phylogenetic groups as reported by Liu, et al. ${ }^{59}$ are indicated (groups I, II, IV, and V) and error bars show SE of four biological replicates.

correlation with the expression of MYB10 (Fig. 3) and is a key step in the anthocyanin pathway, being one of the late biosynthetic enzymes. BBX1 was chosen because of its gene expression profile, both in the fruit development and diurnal rhythm experiments as well as its ability to activate the MYB10 promoter. Infiltrations were 
(A)

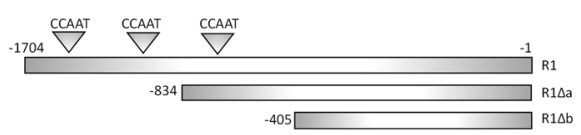

(B)

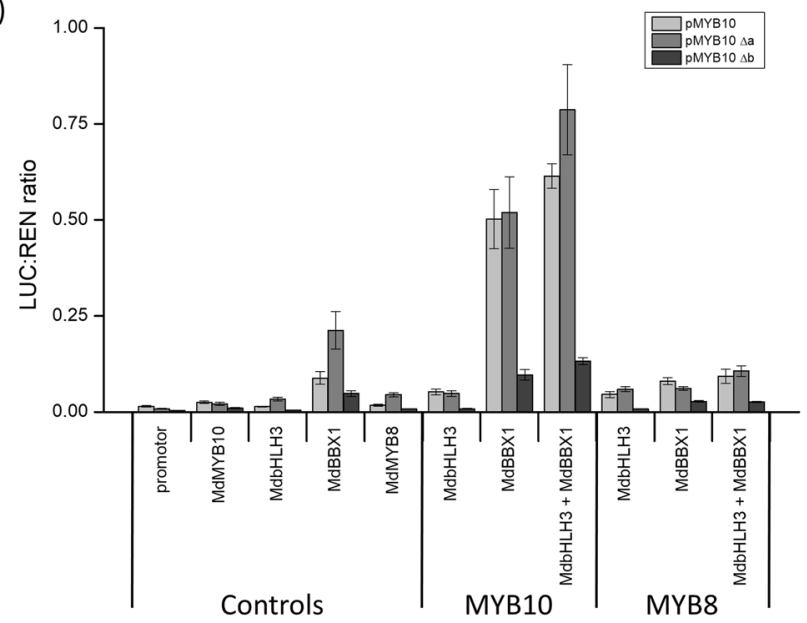

Figure 5. Identification of the region of the MYB10 promoter activated by BBX1. (A) Schematic of the full length and two truncated versions $(\triangle \mathrm{a}$ and $\triangle \mathrm{b}$ ) of the $M Y B 10$ promoter used to identify the region sensitive to the presence of BBX1. Position of three CCAAT motifs indicated. (B) Transient expression assay using the full length $M Y B 10$ promoter and the two truncated versions. Full length and truncated promoters were run alone, with MYB10, and with MYB8 to test activation capability of BBX1 with and without a bHLH cofactor. Error bars show SE of four biological replicates.

performed in N. benthamiana using the dual luciferase assay (Fig. 6). Infiltration of BBX1 alone did not activate the DFR promoter. Co-infiltration of both MYB10 and bHLH3 resulted in strong activation and this was further strengthened by the addition of BBX1. We checked the DFR promoter sequence for CCAAT motifs, finding two, one at -425 and the other at -659 upstream of the start site.

Over-expression of BBX1 in transgenic 'Royal Gala' apple. The apple tree cultivar 'Royal Gala' was transformed with the $B B X 1 \mathrm{cDNA}$ driven by the CaMV35S promoter. Trees were grown in the glasshouse until sufficiently mature to flower. Apple fruit from five independent transgenic lines were harvested and phenotypically assessed 125 DAFB (Fig. 7A). HPLC analysis confirmed that the concentration of cyanidin-3-O-galctoside varied in the different lines, but mainly showed a reduction compared with the control (Fig. 7B). This suggests that other partners of this transcription factor are required for activation. The over-expression of $B B X 1$ in transgenic fruit was confirmed by RT-qPCR and was shown to be highly elevated in all the lines tested compared with the control (Fig. 7C). However, overexpression of BBX1 did not result in more anthocyanin accumulation in the fruit skin of transgenic apples. Fruit weight, firmness and soluble solids content, expressed in ${ }^{\circ} \mathrm{Brix}$ values were also recorded, showing a significant increase of firmness and soluble solids content in the over-expression lines compared with those in the 'Royal Gala' controls (Supplementary Fig. 4). Ethylene concentration and transcript abundance for MYB10,DFR, and ethylene pathway genes ACS and ACS were also assessed (Supplementary Fig. 5). Other polyphenolics in the over-expression lines were generally similar to those found in 'Royal Gala', although some differences were observed, such as an increase in chlorogenic acid (Supplementary Table 3 ).

\section{Discussion}

In this study the putative role of $B B X$ genes on anthocyanin accumulation in apple was assessed. This family of transcription factors control diverse processes in plants with at least one, BBX33, having a direct role in anthocyanin accumulation in apple fruit skin ${ }^{7}$. To study the $B B X$ family we tested gene expression through fruit development and examined the possible role of a number of $B B X$ genes in directly activating anthocyanin biosynthesis. Diurnal rhythms of candidate $B B X$ genes were measured and $B B X 1$ was chosen to advance into stable transgenic work after showing the ability to activate $M Y B 10$ and DFR.

$B B X$ transcriptional levels vary during fruit apple development. Anthocyanin accumulation in apple skin generally occurs late in fruit development and is a result of the anthocyanin-regulating MYB induction of the genes encoding anthocyanin biosynthetic enzymes. The expression patterns of a subset of $B B X$ genes was assessed during fruit development and compared with both the anthocyanin content and the expression of DFR, UFGT and MYB10. This showed that BBX genes have a range of expression patterns, some of which increased with anthocyanin accumulation in fruit skin, similar to banana $B B X$ expression during fruit development ${ }^{80}$. A number of these genes showed an expression patterns correlating with anthocyanin induction, including in particular $B B X 1,15,33,51$ and 54 . $B B X 1$ showed the highest expression outside group IV $B B X$ s. $B B X 15,51$ and 54 showed increasing expression during development, peaking during the period of highest anthocyanin accumulation, and these are all in group IV, which contains $B B X 33$, already shown to activate the anthocyanin pathway in 


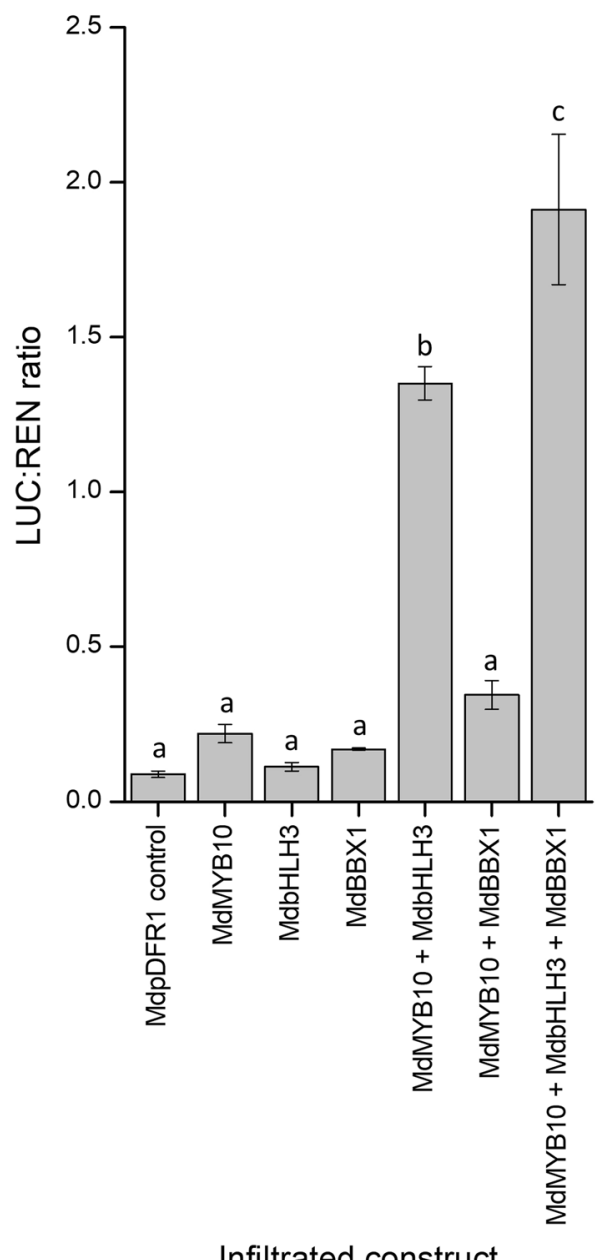

Figure 6. Transient expression assay of the activation of the promoter of the anthocyanin biosynthetic gene DFR by BBX1 in the presence of MYB10 and bHLH3. Error bars show SE of four technical replicates. Differences between averages were statistically analysed using ANOVA and pairwise comparison performed using Fisher's LSD test at the 5\% significance level. Error bars show SE of four biological replicates.

apple 7 . In Arabidopsis, group IV BBXs, in particular, have been identified as linked to light response and so were of interest to assess for any diurnal rhythms they might possess ${ }^{81}$.

The expression pattern of $B B X$ genes demonstrate a diurnal rhythm. BBX genes have been shown to be diurnally regulated in banana and potato ${ }^{80,82}$. In apple, expression analysis over a 48-hour period late in fruit development was analysed (Fig. 2). Strong patterns in expression were seen for GI, light, and temperature-related genes in fruit skin, and these daily rhythms appeared stable in the orchard-grown fruit. $B B X 1,15,33$ and 51 were chosen based on their expression patterns during fruit development. All four BBXs displayed diurnal patterns of expression for both seasons, except for $B B X 33$, which displayed no obvious pattern for season 1 . BBX33 influences anthocyanin in apple ${ }^{7}$ and appears to have been influenced by an unknown factor in season 1 that did not affect $B B X 1, B B X 15$ or $B B X 51$. The diurnal variation in $B B X$ transcription recorded here signifies the importance of sampling times for $B B X \mathrm{~s}$, and indeed all environmentally regulated genes, when considering experimental design. For example, results varied as much as six-fold for $B B X 1$ between sampling at $0800 \mathrm{~h}$ or $2000 \mathrm{~h}$.

The expression of anthocyanin-related genes demonstrates a clear diurnal rhythm, co-ordinated by MYB10. The diurnal rhythm demonstrated by a number of the $B B X$ genes suggests that diurnal rhythms may also be important in anthocyanin regulation. We tested key genes in the anthocyanin pathway, and showed that transcript accumulation of these anthocyanin biosynthetic genes followed a very similar pattern over the 48-hour sampling period (Fig. 3). This was also evident for the MYB regulator, MYB10, confirmed by statistical analysis. This suggests that the pathway genes are modulated in a highly controlled manner, although further work is necessary to examine the interplay of the transcriptional control and environmental factors determining this phenomenon. We did not find any correlation between expression patterns of MYB10 and $B B X 1,15,33$ or 51 (Supplementary Table $2 A$ ). Interestingly, $B B X 1$ did have a significant correlation with temperature (Supplementary Fig. 2B), a relationship supported by previous findings $\mathrm{s}^{7,32}$. Temperature readings during the sampling period are provided in Supplementary Fig. 6. Furthermore, BBX33 transcription had a significant 
(A)
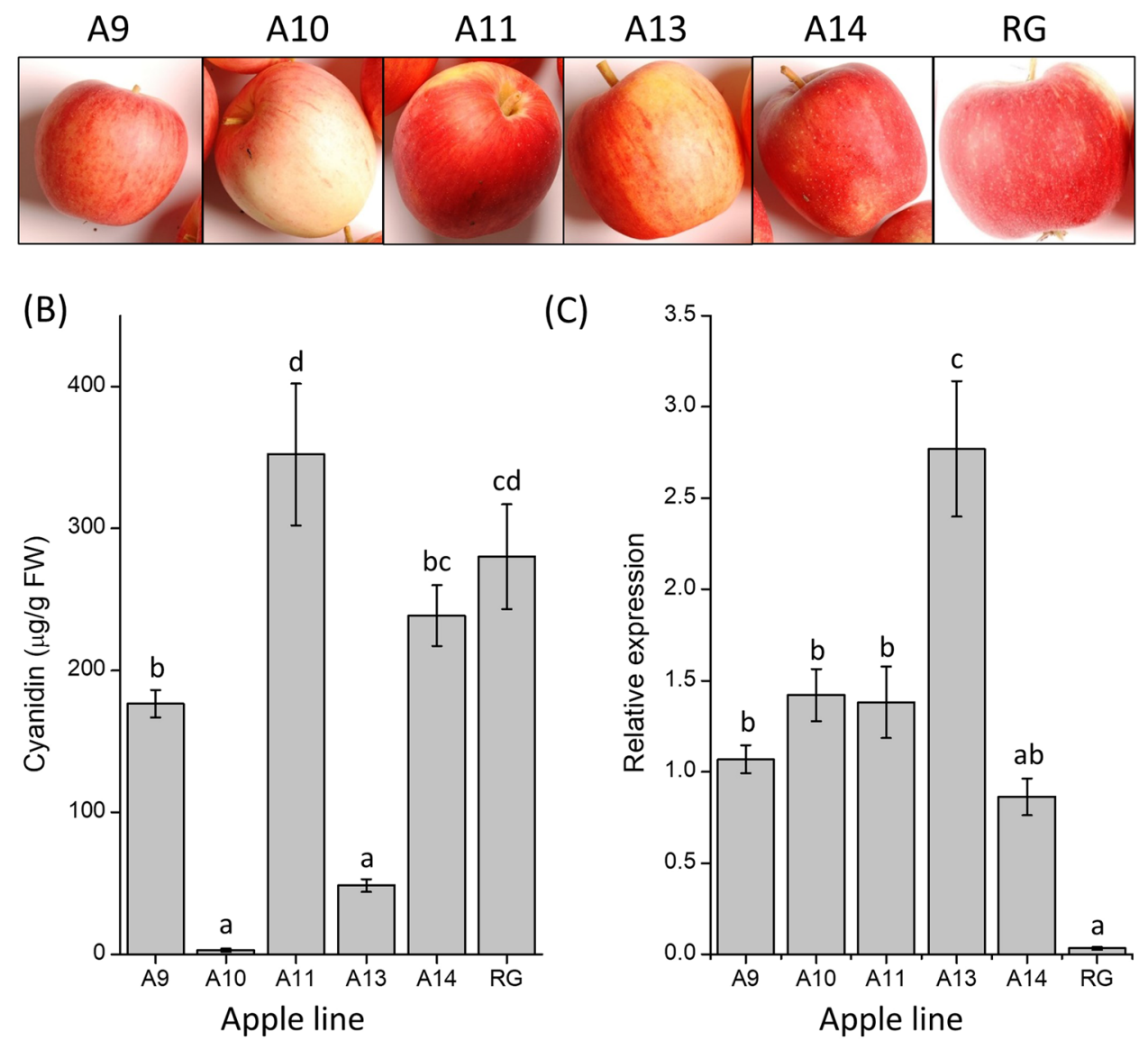

(C)

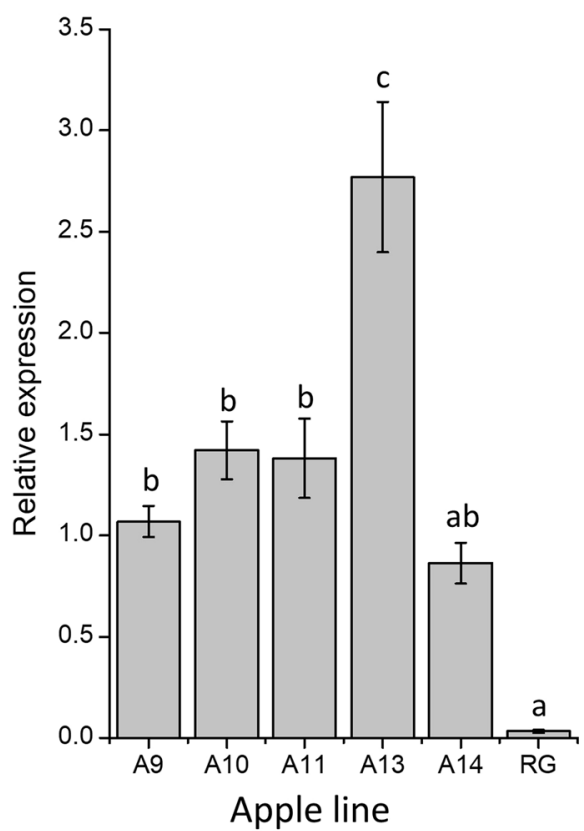

Figure 7. Characterisation of 35S:BBX1 transgenic lines. (A) Images of representative apples from each of the lines. (B) Cyanidin concentration in fruit skin for the transgenic lines compared with 'Royal Gala' control (RG). Cyanidin concentrations reported are the sums of cyanidin 3-O-galactoside, cyanidin 3-O-glucoside, and cyanidin 3-O-arabinoside in $\mu \mathrm{g} / \mathrm{g}$ FW. Cyanidin 3-O-galactoside accounted for over $90 \%$ in every sample tested. (C) Gene expression of BBX1 in the 35S:BBX1 transgenic lines compared with that in 'Royal Gala' control fruit (RG). Results are average expression of three biological replicates and four technical replicates relative to Actin. Error bars show SE of four biological replicates. Differences between averages were statistically analysed using ANOVA and pairwise comparison performed using Fisher's LSD test at the 5\% significance level.

correlation with HY5 transcription for season 1, supporting the model that BBX33 influences anthocyanin via HY5 proposed by Bai et al. ${ }^{7}$ (Supplementary Table 2C). This correlation, however, was not seen for season 2 and so appears to vary seasonally and geographically.

BBX genes can directly activate the apple anthocyanin-regulating MYB. To further determine the role of $B B X$ s, we tested different sub-groups of the $B B X$ family for transactivation of the promoter of the anthocyanin regulator MYB10 using luciferase assays. Activation capability was not specific to particular groups. As $B B X 33$ has been published by Bai et al. ${ }^{7}$ and group IV has been reported as important in light signalling ${ }^{81}$, we focused on this group by testing more of its members. There was substantial variation in the effect of addition of $M Y B 10$ and $b H L H 3$; for example, $B B X 15$ had little activation without $M Y B 10$ and $b H L H 3$, whereas $B B X 17$ had the same degree of activation regardless of the presence of $M Y B 10$ or $b H L H 3$. The highest activating $B B X$ s were $B B X 1,35,15,51$ and 54 , to which the closest predicted amino acid (AA) BLAST hits in Arabidopsis were AtBBX5 (AtCOL4), AtBBX12, AtBBX21, and AtBBX24, respectively (with apple BBX51 and 54 both being most similar to AtBBX24). In Arabidopsis these BBXs have been reported to be involved with photosynthesis, photomorphogenesis, and various stress responses ${ }^{57,83}$. AtBBX21 and AtBBX24 specifically have been linked to the regulation of $H Y 5^{84}$, which is supported by results here that showed developmental transcription, diurnal rhythm, and MYB10 activation for the apple homologues BBX15 and BBX51. Compared with the highest activating BBXs, $B B X 33$ did not highly activate $M Y B 10$, which, being involved in anthocyanin accumulation, suggests a low threshold is required for BBX activation of the anthocyanin pathway. This ability of BBXs to activate MYB10 suggests the presence of cis elements in the MYB10 promoter that might be bound by BBX proteins.

A BBX-related cis element is necessary for BBX transactivation of the MYB10 promoter. Data from the promoter deletion study (Fig. 5) showed that BBX activation of the MYB10 promoter is partly dependent 
on the presence of upstream sequences. The shortest promoter fragment, $\Delta \mathrm{b}$, was not able to activate the reporter to the same extent as either the full promoter tested or $\triangle \mathrm{a}$. This suggests that BBX1 requires a motif that is absent in the most truncated version. Previous research on CONSTANS binding with the FLOWERING LOCUS T (FT) promoter has identified two possible motifs in Arabidopsis. A CCAAT box binding domain was identified by Wenkel et al. ${ }^{78}$, and this relatively common eukaryotic cis-element binds with the NF-Y/HAP complex to drive transcription. The full-length MYB10 promoter showed a high level of transactivation in the presence of BBX1 (Fig. 5B). There are three CCAAT cis-elements in the 5 ' region of this promoter sequence (Fig. 5A). There was no significant reduction when the same assay was performed using the truncated $\Delta$ a promoter fragment. This contains just one CCAAT domain. However, when $\triangle \mathrm{b}$ containing no CCAAT motifs was used, there was a dramatic reduction in activation. This suggests the region between $M Y B 10 \Delta a$ and $M Y B 10 \Delta b$ is crucial for the effects of BBX1 and indicates that the CCAAT motif may be essential for BBX binding. However, there was still some activation, indicating that another element may be present. Bai et al. ${ }^{7}$ reported activation of $M Y B 10$ also being influenced by ACGT-containing elements and Polymorphic G-boxes, and found activation occurring down to $\mathrm{a}-314 \mathrm{bp}$ promotor fragment, suggesting a possible binding site between $-405 \mathrm{bp}$ and $-314 \mathrm{bp}$. PLACE (Plant cis-acting regulatory DNA elements) analysis for this region identified many potential sites, but no CCAAT, ACGT-containing elements, or G-boxes. A second, more specific element has been described for CO binding of the FT promoter ${ }^{85}$. This sequence was identified as TGTG(N2-3)ATG, but was not found in the MYB10 promoter. As well as the MYB10 promotor, light-induced factors could also be upregulating the anthocyanin biosynthetic genes directly by binding their promotors.

$B B X 1$ in combination with $M Y B 10$ and $b H L H 3$ activates the DFR promotor. Previous results indicated that light responsive regulators of anthocyanin could be acting on the structural genes in the pathway directly $^{38,86}$. Transient expression assays were performed on the promotor of $D F R$ to test this for $B B X 1$. BBX1 did not display a significant activation of the DFR promotor when infiltrated alone (Fig. 6). As expected, a strong activation was seen when the DFR promoter was infiltrated with $M Y B 10$ and $b H L H 3$, which was enhanced with the co-infiltration of $B B X 1$, suggesting some combinatorial effect of members of the MBW complex and BBX1. $B B X 33$ has previously been shown to be regulated by HY5 and to regulate MYB10 ${ }^{7}$.

$B B X 1$ overexpression in apple may affect fruit maturation, but did not increase anthocyanin accumulation. BBX1 was over-expressed in apple trees. Fruit weight was not significantly different between the transgenic lines and the 'Royal Gala' control. There was, however, a significant difference in fruit firmness and soluble solids content (Supplementary Fig. 4). Ethylene was also significantly lower in the transgenic fruit (Supplementary Fig. 5A), which alongside the difference in firmness and soluble solids content, suggests an effect on fruit maturity. Transcript abundance for ethylene genes did not show the same pattern as ethylene measurements, which were all reduced in the over-expressing lines, suggesting the control of other factors (Supplementary Fig. 5B). BBXs have been linked to regulating shade avoidance, which is controlled by hormones that influence plant growth, such as ethylene ${ }^{87}$. The anthocyanin content of the transgenic fruit was either similar to control fruit, as shown in the independent lines A11 and A14, or reduced as shown in A9, A10, A13 (Fig. 6B). Although the expression of $B B X 1$ was high compared with that in the 'Royal Gala' control (Fig. 6C), the expression levels of the other genes tested were more similar to MYB10 than to BBX1 (Supplementary Fig. 5B), indicating that the over-expression did not overcome the control $M Y B 10$ has over the anthocyanin pathway, particularly as the anthocyanin content correlated to the transcript abundance of MYB10. Other environmental conditions, such as temperature, are likely to contribute to the results of this study. It is possible that thresholds of temperature sensitive expression of $B B X 1$ were not reached during this work, and therefore anthocyanin accumulation was not affected. Future experiments could test this by manipulating temperature and measuring $B B X 1$ expression along with anthocyanin concentration.

\section{Conclusion}

$B B X$ genes play a diverse set of roles in plants. The few reports to date have classified the family and focused on individual $B B X \mathrm{~s}^{7,59}$. Here, a wide range of $B B X$ s were scrutinised to determine their potential as activators of the anthocyanin pathway, identifying candidates worth pursuing. The expression of a number of $B B X$ s correlated with cyanidin-3-O-galactoside during fruit development. $B B X 1$ activated the promoters of $M Y B 10$ and $D F R$; however, when $B B X 1$ was over-expressed in apple, there was no apparent increase in anthocyanin. Taken together, this evidence suggests that there may be an element of control of the anthocyanin pathway by $B B X$ s with other partner transcription factors. Identifying transcriptional partners that influence colour in apple alongside the previously demonstrated effect of $B B X 33^{7}$ will aid the development of apple varieties with predictable colour development.

Received: 3 January 2019; Accepted: 31 October 2019;

Published online: 28 November 2019

\section{References}

1. Boyer, J. \& Liu, R. H. Apple phytochemicals and their health benefits. Nutrition Journal 3, 1-15 (2004).

2. Espley, R. \& Martens, S. In Bioactives in Fruit 81-100 (John Wiley \& Sons, Ltd, 2013).

3. He, J. \& Giusti, M. M. Anthocyanins: Natural Colorants with Health-Promoting Properties. Annual Review of Food Science and Technology 1, 163-187 (2010).

4. Scalbert, A. \& Williamson, G. Dietary Intake and Bioavailability of Polyphenols. The Journal of Nutrition 130 (2000).

5. Cliff, M., Sanford, K., Wismer, W. \& Hampson, C. Use of Digital Images for Evaluation of Factors Responsible for Visual Preference of Apples by Consumers. HortScience 37, 1127-1131 (2002).

6. Marjorie, C. \& Margaret, C. Development of a model for prediction of consumer liking from visual attributes of new and established apple cultivars. Journal of American Pomological Society 56, 223 (2002). 
7. Bai, S. et al. An apple B-box protein, MdCOL11, is involved in UV-B- and temperature-induced anthocyanin biosynthesis. Planta 240, 1051-1062 (2014).

8. Delgado-Pelayo, R., Gallardo-Guerrero, L. \& Hornero-Méndez, D. Chlorophyll and carotenoid pigments in the peel and flesh of commercial apple fruit varieties. Food Research International 65, Part B, 272-281 (2014).

9. Lancaster, J. E., Grant, J. E., Lister, C. E. \& Taylor, M. C. Skin Color in Apples - Influence of Copigmentation and Plastid Pigments on Shade and Darkness of Red Color in Five Genotypes. Journal of the American Society for Horticultural Science 119, 63-69 (1994).

10. Li, P. \& Cheng, L. The elevated anthocyanin level in the shaded peel of 'Anjou' pear enhances its tolerance to high temperature under high light. Plant Science 177, 418-426 (2009).

11. Steyn, W. J., Wand, S. J. E., Holcroft, D. M. \& Jacobs, G. Anthocyanins in vegetative tissues: a proposed unified function in photoprotection. New Phytologist 155, 349-361 (2002).

12. Felicetti, D. A. \& Schrader, L. E. Changes in pigment concentrations associated with the degree of sunburn browning of 'Fuji' apple. Journal of the American Society for Horticultural Science 133, 27-34 (2008).

13. Gould, K. \& Lister, C. In Flavonoids 397-441 (CRC Press, 2005).

14. Gonzalez, A., Zhao, M., Leavitt, J. M. \& Lloyd, A. M. Regulation of the anthocyanin biosynthetic pathway by the TTG1/bHLH/Myb transcriptional complex in Arabidopsis seedlings. The Plant Journal 53, 814-827 (2008).

15. Albert, N. et al. A Conserved Network of Transcriptional Activators and Repressors Regulates Anthocyanin Pigmentation in Eudicots. Vol. 26 (2014).

16. Henry-Kirk, R. A., McGhie, T. K., Andre, C. M., Hellens, R. P. \& Allan, A. C. Transcriptional analysis of apple fruit proanthocyanidin biosynthesis. Journal of experimental botany 63, 5437-5450 (2012).

17. Baudry, A. et al. TT2, TT8, and TTG1 synergistically specify the expression of BANYULS and proanthocyanidin biosynthesis in Arabidopsis thaliana. The Plant Journal 39, 366-380 (2004)

18. Ravaglia, D. et al. Transcriptional regulation of flavonoid biosynthesis in nectarine (Prunus persica) by a set of R2R3 MYB transcription factors. BMC Plant Biology 13, 68 (2013).

19. Schaart, J. G. et al. Identification and characterization of MYB-bHLH-WD40 regulatory complexes controlling proanthocyanidin biosynthesis in strawberry (Fragaria $\times$ ananassa) fruits. New Phytologist 197, 454-467 (2013).

20. Albert, N. W. Subspecialization of R2R3-MYB Repressors for Anthocyanin and Proanthocyanidin Regulation in Forage Legumes. Frontiers in Plant Science 6 (2015).

21. Nemesio-Gorriz, M. et al. Identification of Norway Spruce MYB-bHLH-WDR Transcription Factor Complex Members Linked to Regulation of the Flavonoid Pathway. Frontiers in plant science 8, 305 (2017).

22. Espley, R. et al. Red colouration in apple fruit is due to the activity of the MYB transcription factor, MdMYB10. The Plant Journal 49, 414-427 (2007).

23. Li, S. Transcriptional control of flavonoid biosynthesis: Fine-tuning of the MYB-bHLH-WD40 (MBW) complex. Plant Signaling \& Behavior 9, e27522 (2014)

24. Takos, A. et al. Light-induced expression of a MYB gene regulates anthocyanin biosynthesis in red apples. Plant Physiol 142, 1216-1232 (2006).

25. Ban, Y. et al. Isolation and functional analysis of a MYB transcription factor gene that is a key regulator for the development of red coloration in apple skin. Plant Cell Physiol 48, 958-970 (2007).

26. Allan, A., Hellens, R. \& Laing, W. MYB transcription factors that colour our fruit. Trends Plant Sci 13, 99-102 (2008).

27. Telias, A. et al. Apple skin patterning is associated with differential expression of MYB10. BMC Plant Biology 11, 93 (2011).

28. Albert, N. W. et al. Members of an R2R3-MYB transcription factor family in Petunia are developmentally and environmentally regulated to control complex floral and vegetative pigmentation patterning. The Plant Journal 65, 771-784 (2011).

29. Matsui, K., Umemura, Y. \& Ohme-Takagi, M. AtMYBL2, a protein with a single MYB domain, acts as a negative regulator of anthocyanin biosynthesis in Arabidopsis. The Plant Journal 55, 954-967 (2008).

30. Dubos, C. et al. MYBL2 is a new regulator of flavonoid biosynthesis in Arabidopsis thaliana. The Plant Journal 55, 940-953 (2008).

31. Aharoni, A. et al. The strawberry FaMYB1 transcription factor suppresses anthocyanin and flavonol accumulation in transgenic tobacco. The Plant Journal 28, 319-332 (2001).

32. Lin-Wang, K. U. I. et al. High temperature reduces apple fruit colour via modulation of the anthocyanin regulatory complex. Plant, Cell \& Environment, 1-15 (2011).

33. Xie, X.-B. et al. The bHLH transcription factor MdbHLH3 promotes anthocyanin accumulation and fruit colouration in response to low temperature in apples. Plant, Cell \&. Environment 35, 1884-1897 (2012).

34. Dong, Y.-H. et al. Expression of pigmentation genes and photo-regulation of anthocyanin biosynthesis in developing Royal Gala apple flowers. Functional Plant Biology 25, 245-252 (1998).

35. Jakopic, J., Stampar, F. \& Veberic, R. The influence of exposure to light on the phenolic content of 'Fuji' apple. Scientia Horticulturae 123, 234-239 (2009).

36. Ban, Y., Honda, C., Bessho, H., Pang, X.-M. \& Moriguchi, T. Suppression subtractive hybridization identifies genes induced in response to UV-B irradiation in apple skin: isolation of a putative UDP-glucose 4-epimerase. Journal of Experimental Botany $\mathbf{5 8}$, 1825-1834 (2007).

37. Henry-Kirk, R. A. et al. Solar UV light regulates flavonoid metabolism in apple (Malus x domestica). Plant, Cell \& Environment 41, 675-688 (2018).

38. Shin, D. H. et al. HY5 regulates anthocyanin biosynthesis by inducing the transcriptional activation of the MYB75/PAP1 transcription factor in Arabidopsis. FEBS Letters 587, 1543-1547 (2013).

39. Zhang, Y., Zheng, S., Liu, Z., Wang, L. \& Bi, Y. Both HY5 and HYH are necessary regulators for low temperature-induced anthocyanin accumulation in Arabidopsis seedlings. Journal of Plant Physiology 168, 367-374 (2011).

40. Chang, C.-sJ. et al. LZF1, a HY5-regulated transcriptional factor, functions in Arabidopsis de-etiolation. The Plant Journal 54, 205-219 (2008)

41. Favory, J. J. et al. Interaction of COP1 and UVR8 regulates UV-B-induced photomorphogenesis and stress acclimation in Arabidopsis. The EMBO Journal 28, 591-601 (2009).

42. Liu, L., Gregan, S., Winefield, C. \& Jordan, B. From UVR8 to flavonol synthase: UV-B-induced gene expression in Sauvignon blanc grape berry. Plant, Cell \& Environment 38, 905-919 (2015).

43. Deng, X.-W., Caspar, T. \& Quail, P. H. cop1: a regulatory locus involved in light-controlled development and gene expression in Arabidopsis. Genes \& Development 5, 1172-1182 (1991).

44. Li, Y.-Y. et al. MdCOP1 Ubiquitin E3 Ligases Interact with MdMYB1 to Regulate Light-Induced Anthocyanin Biosynthesis and Red Fruit Coloration in Apple. Plant Physiology 160, 1011-1022 (2012).

45. Maier, A. et al. Light and the E3 ubiquitin ligase COP1/SPA control the protein stability of the MYB transcription factors PAP1 and PAP2 involved in anthocyanin accumulation in Arabidopsis. The Plant Journal 74, 638-651 (2013).

46. Datta, S. et al. LZF1/SALT TOLERANCE HOMOLOG3, an Arabidopsis B-Box Protein Involved in Light-Dependent Development and Gene Expression, Undergoes COP1-Mediated Ubiquitination. The Plant Cell 20, 2324-2338 (2008).

47. Hayama, R. \& Coupland, G. The Molecular Basis of Diversity in the Photoperiodic Flowering Responses of Arabidopsis and Rice. Plant Physiology 135, 677-684 (2004).

48. Putterill, J., Robson, F., Lee, K., Simon, R. \& Coupland, G. The CONSTANS gene of arabidopsis promotes flowering and encodes a protein showing similarities to zinc finger transcription factors. Cell 80, 847-857 (1995). 
49. Valverde, F. et al. Photoreceptor Regulation of CONSTANS Protein in Photoperiodic Flowering. Science 303, 1003 (2004).

50. Onouchi, H., Igeño, M. I., Périlleux, C., Graves, K. \& Coupland, G. Mutagenesis of Plants Overexpressing CONSTANS Demonstrates Novel Interactions among Arabidopsis Flowering-Time Genes. The Plant Cell 12, 885-900 (2000).

51. Samach, A. et al. Distinct Roles of CONSTANS Target Genes in Reproductive Development of Arabidopsis. Science 288, 1613 (2000).

52. Borden, K. L. B. RING domains: master builders of molecular scaffolds?1. Journal of Molecular Biology 295, 1103-1112 (2000).

53. Crocco, C. D. \& Botto, J. F. BBX proteins in green plants: Insights into their evolution, structure, feature and functional diversification. Gene 531, 44-52 (2013).

54. Strayer, C. et al. Cloning of the Arabidopsis clock gene TOC1, an autoregulatory response regulator homolog. Science 289, 768 (2000).

55. Gangappa, S. N. \& Botto, J. F. The BBX family of plant transcription factors. Trends in Plant Science 19, 460-470 (2014).

56. Robson, F. et al. Functional importance of conserved domains in the flowering-time gene CONSTANS demonstrated by analysis of mutant alleles and transgenic plants. The Plant Journal 28, 619-631 (2001).

57. Khanna, R. et al. The Arabidopsis B-Box Zinc Finger Family. The Plant Cell 21, 3416-3420 (2009).

58. Huang, J., Zhao, X., Weng, X., Wang, L. \& Xie, W. The Rice B-Box Zinc Finger Gene Family: Genomic Identification, Characterization, Expression Profiling and Diurnal Analysis. PLoS ONE 7, e48242 (2012).

59. Liu, X., Li, R., Dai, Y., Chen, X. \& Wang, X. Genome-wide identification and expression analysis of the B-box gene family in the Apple (Malus domestica Borkh.) genome. Molecular Genetics and Genomics (2017).

60. Jeong, D.-H., Sung, S.-K. \& An, G. Molecular cloning and characterization of constans-like cDNA clones of the fuji apple. Journal of Plant Biology 42, 23-31 (1999).

61. Lagercrantz, U. \& Axelsson, T. Rapid Evolution of the Family of CONSTANS LIKE Genes in Plants. Molecular Biology and Evolution 17, 1499-1507 (2000)

62. Jin, J. et al. PlantTFDB 4.0: toward a central hub for transcription factors and regulatory interactions in plants. Nucleic Acids Research 45, D1040-D1045 (2017).

63. Simon, S., Rühl, M., de Montaigu, A., Wötzel, S. \& Coupland, G. Evolution of CONSTANS Regulation and Function after Gene Duplication Produced a Photoperiodic Flowering Switch in the Brassicaceae. Molecular Biology and Evolution 32, 2284-2301 (2015).

64. Ledger, S., Strayer, C., Ashton, F., Kay, S. A. \& Putterill, J. Analysis of the function of two circadian-regulated CONSTANS-LIKE genes. The Plant Journal 26, 15-22 (2001).

65. Suarez-Lopez, P. et al. CONSTANS mediates between the circadian clock and the control of flowering in Arabidopsis. Nature 410, $1116-1120(2001)$.

66. Hellens, R. et al. Transient expression vectors for functional genomics, quantification of promoter activity and RNA silencing in plants. Plant Methods 1, 13 (2005).

67. Espley, R. et al. Multiple repeats of a promoter segment causes transcription factor autoregulation in red apples. Plant Cell 21, 168-183 (2009).

68. Lin-Wang, K. et al. An R2R3 MYB transcription factor associated with regulation of the anthocyanin biosynthetic pathway in Rosaceae. BMC Plant Biology 10, 50 (2010).

69. Dare, A. P. et al. Silencing a phloretin-specific glycosyltransferase perturbs both general phenylpropanoid biosynthesis and plant development. The Plant Journal 91, 237-250 (2017).

70. Johnston, J. W., Gunaseelan, K., Pidakala, P., Wang, M. \& Schaffer, R. J. Co-ordination of early and late ripening events in apples is regulated through differential sensitivities to ethylene. Journal of Experimental Botany 60, 2689-2699 (2009).

71. R Core Team. R: A language and environment for statistical computing. R Foundation for Statistical Computing, Vienna, Austria. URL, https://www.R-project.org/ (2016).

72. Hayter, A. J. The Maximum Familywise Error Rate of Fisher's Least Significant Difference Test. Journal of the American Statistical Association 81, 1000-1004 (1986).

73. Yao, J.-L., Cohen, D., Atkinson, R., Richardson, K. \& Morris, B. Regeneration of transgenic plants from the commercial apple cultivar'Royal Gala'. Plant Cell Reports 14, 407-412 (1995).

74. Fowler, S. et al. GIGANTEA: A circadian clock-controlled gene that regulates photoperiodic flowering in Arabidopsis and encodes a protein with several possible membrane-spanning domains. EMBO Journal 18, 4679-4688 (1999).

75. Miller, T. A., Muslin, E. H. \& Dorweiler, J. E. A maize CONSTANS-like gene, conz1, exhibits distinct diurnal expression patterns in varied photoperiods. Planta 227, 1377-1388 (2008).

76. Dong, M. A., Farré, E. M. \& Thomashow, M. F. Circadian Clock-Associated 1 And Late Elongated Hypocotyl Regulate Expression Of The C-Repeat Binding Factor (CBF) pathway in Arabidopsis. Proceedings of the National Academy of Sciences 108, 7241-7246 (2011).

77. Giorno, F., Guerriero, G., Baric, S. \& Mariani, C. Heat shock transcriptional factors in Malus domestica: identification, classification and expression analysis. BMC Genomics 13, 1-13 (2012).

78. Wenkel, S. et al. CONSTANS and the CCAAT box binding complex share a functionally important domain and interact to regulate flowering of Arabidopsis. The Plant Cell Online 18, 2971-2984 (2006).

79. Gnesutta, N. et al. CONSTANS imparts DNA sequence-specificity to the histone-fold NF-YB/NF-YC dimer. The Plant Cell 29, 1516-1532 (2017).

80. Chaurasia, A. K. et al. Molecular characterization of CONSTANS-Like (COL) genes in banana. Physiology and Molecular Biology of Plants 22, 1-15 (2016).

81. Sarmiento, F. The BBX subfamily IV. Plant Signaling \& Behavior 8 (2013)

82. Talar, U., Kiełbowicz-Matuk, A., Czarnecka, J. \& Rorat, T. Genome-wide survey of B-box proteins in potato (Solanum tuberosum)Identification, characterization and expression patterns during diurnal cycle, etiolation and de-etiolation. PLoS One 12, e0177471 (2017).

83. Ji-Hee, M., Jung-Sung, C., Kyeong-Hwan, L. \& Soo, K. C. The CONSTANS-like 4 transcription factor, AtCOL4, positively regulates abiotic stress tolerance through an abscisic acid-dependent manner in Arabidopsis. Journal of Integrative Plant Biology 57, 313-324 (2015).

84. Yadukrishnan, P., Job, N., Johansson, H. \& Datta, S. Opposite Roles of Group IV BBX Proteins: Exploring Missing Links between Structural and Functional Diversity. Plant Signaling \& Behavior, 1-13 (2018).

85. Tiwari, S. B. et al. The flowering time regulator CONSTANS is recruited to the FLOWERING LOCUS T promoter via a unique ciselement. New Phytologist 187, 57-66 (2010).

86. Chattopadhyay, S., Ang, L.-H., Puente, P., Deng, X.-W. \& Wei, N. Arabidopsis bZIP Protein HY5 Directly Interacts with LightResponsive Promoters in Mediating Light Control of Gene Expression. The Plant Cell 10, 673-683 (1998).

87. Crocco, C. D. et al. The transcriptional regulator BBX24 impairs DELLA activity to promote shade avoidance in Arabidopsis thaliana. Nature Communications 6, 6202 (2015). 


\section{Acknowledgements}

We are grateful to Monica Dragulescu and glasshouse staff for management of plants and to Geeta Chhiba for providing media and buffers. We also thank Tony McGhie for help with HPLC. KM was funded by the Finnish horticultural foundation Maiju ja Yrjö Rikalan Puutarhasäätiö.

\section{Author contributions}

B.P., R.E., J.P. and A.A. contributed to project planning; A.D. conducted HPLC analysis; R.E., R.H., K.M. and B.P. performed RT-PCR; A.F. and R.D. collected plant material; B.P. conducted statistical analysis; K.M., S.H. and B.P. performed transient assays; S.T. generated transgenic apple lines; B.P., R.E., J.P. and A.A. wrote the manuscript; All authors contributed to editing the manuscript.

\section{Competing interests}

The authors declare no competing interests.

\section{Additional information}

Supplementary information is available for this paper at https://doi.org/10.1038/s41598-019-54166-2.

Correspondence and requests for materials should be addressed to A.C.A.

Reprints and permissions information is available at www.nature.com/reprints.

Publisher's note Springer Nature remains neutral with regard to jurisdictional claims in published maps and institutional affiliations.

Open Access This article is licensed under a Creative Commons Attribution 4.0 International License, which permits use, sharing, adaptation, distribution and reproduction in any medium or format, as long as you give appropriate credit to the original author(s) and the source, provide a link to the Creative Commons license, and indicate if changes were made. The images or other third party material in this article are included in the article's Creative Commons license, unless indicated otherwise in a credit line to the material. If material is not included in the article's Creative Commons license and your intended use is not permitted by statutory regulation or exceeds the permitted use, you will need to obtain permission directly from the copyright holder. To view a copy of this license, visit http://creativecommons.org/licenses/by/4.0/.

(C) The Author(s) 2019 